\title{
Involvement of the adaptor protein 3 complex in lignocellulase secretion in Neurospora crassa revealed by comparative genomic screening
}

Xue Pei ${ }^{1,2+}$, Feiyu Fan ${ }^{2 \dagger}$, Liangcai Lin ${ }^{2}$, Yong Chen ${ }^{2}$, Wenliang Sun ${ }^{2}$, Shihong Zhang ${ }^{1 *}$ and Chaoguang Tian ${ }^{2 *}$

\begin{abstract}
Background: Lignocellulase hypersecretion has been achieved in industrial fungal workhorses such as Trichoderma reesei, but the underlying mechanism associated with this process is not well understood. Although previous comparative genomic studies have revealed that the mutagenic T. reesei strain RUT-C30 harbors hundreds of mutations compared with its parental strain QM6a, how these mutations actually contribute to the hypersecretion phenotype remains to be elucidated.
\end{abstract}

Results: In this study, we systematically screened gene knockout $(K O)$ mutants in the cellulolytic fungus Neurospora crassa, which contains orthologs of potentially defective T. reesei RUT-C30 mutated genes. Of the 86 deletion mutants screened in N. crassa, 12 exhibited lignocellulase production more than 25\% higher than in the wild-type (WT) strain and 4 showed nearly 25\% lower secretion. We observed that the deletion of Ncap3m (NCU03998), which encodes the $\mu$ subunit of the adaptor protein 3 (AP-3) complex in N. crassa, led to the most significant increase in lignocellulase secretion under both Avicel and xylan culture conditions. Moreover, strains lacking the $\beta$ subunit of the AP-3 complex, encoded by Ncap3b (NCU06569), had a similar phenotype to $\Delta$ Ncap3m, suggesting that the AP-3 complex is involved in lignocellulase secretion in N. crassa. We also found that the transcriptional abundance of major lignocellulase genes in $\Delta$ Ncap3m was maintained at a relatively higher level during the late stage of fermentation compared with the WT, which might add to the hypersecretion phenotype. Finally, we found that importation of the T. reesei ap $3 m$ ortholog Trap3m into $\Delta N c a p 3 m$ can genetically restore secretion of lignocellulases to normal levels, which suggests that the effect of the AP-3 complex on lignocellulase secretion is conserved in cellulolytic ascomycetes.

Conclusions: Using the model cellulolytic fungus N. crassa, we explored potential hypersecretion-related mutations in T. reesei strain RUT-C30. Through systematic genetic screening of 86 corresponding orthologous $\mathrm{KO}$ mutants in $\mathrm{N}$. crassa, we identified several genes, particularly those encoding the AP-3 complex that contribute to lignocellulase secretion. These findings will be useful for strain improvement in future lignocellulase and biomass-based chemical production.

Keywords: Trichoderma reesei, RUTC30, Neurospora crassa, Adaptor protein 3 complex, Lignocellulase secretion

\footnotetext{
*Correspondence: zhang_sh@jlu.edu.cn; tian_cg@tib.cas.cn

${ }^{\dagger}$ Xue Pei and Feiyu Fan contributed equally to this work

${ }^{1}$ College of Plant Sciences, Jilin University, Changchun 130062, China

${ }^{2}$ Key Laboratory of Systems Microbial Biotechnology, Tianjin Institute

of Industrial Biotechnology, Chinese Academy of Sciences, Tianjin 300308,

China

Full list of author information is available at the end of the article
} 


\section{Background}

In recent years, the use of large quantities of inexpensive plant biomass as feedstock for biofuel production has become an increasing focus of research. One of the key steps of the integrated biomass conversion process is production of lignocellulases, which are responsible for the degradation of plant cell wall material into fermentable sugars. This step is one of the major determinants of biofuel production costs [1, 2]. In nature, saprophytic fungi have evolved a highly efficient capability to secrete lignocellulases, with this property subsequently exploited in industry for lignocellulase production [3, 4].

Lignocellulases are synthesized and secreted through fungal secretory pathways $[5,6]$. Generally, the nascent peptides of lignocellulases must be translocated into the endoplasmic reticulum (ER) lumen for folding and modification before delivery to the Golgi apparatus for further processing and subsequent targeting to their final proper destination (e.g., the extracellular matrix) via small secretory vesicles $[7,8]$. To balance intracellular nutritional homeostasis or relieve the deleterious effects of unfolded or misfolded proteins, lignocellulases may undergo turnover via proteasome- or vacuole/lysosome-mediated degradation processes to meet the demand of nitrogen source recycling within fungal cells $[9,10]$. For this reason, the dynamic competition between intracellular protein degradation and secretion may determine the final production titer of lignocellulases.

Currently, the most successful industrial hosts for lignocellulase production are primarily those originating from Trichoderma species, for example, T. reesei RUT-C30 (ATCC 56765). This hypersecretion mutant was obtained in the late 1970s via a three-step procedure [11-14]: (1) UV mutagenesis of wild-type (WT) Qm6a to generate isolate M7; (2) creation of NG14 by further mutagenesis of $\mathrm{M} 7$ using $\mathrm{N}$-nitroguanidine; and (3) selection of RUT-C30 following another UV round of mutagenesis on the NG14 strain. Although the final secretion titer has been increased 20-fold in RUT-C30 $[11,15]$, little is known about the mechanism underlying the physiological hypersecretion process, especially that responsible for remodeling of the secretory pathway and associated regulation. With advances in omics technologies, recent comparative genomic studies have revealed that RUT-C30 contains a large chromosomal fragment deletion and hundreds of small mutations compared with its paternal strain QM6a. Several mutations have affected genes involved in lignocellulase regulation, such as two previously well-characterized targets: the carbon catabolite repression (CCR) regulator cre1 (tre120117), which was found to be truncated in RUT-C30 [16], and the $g l s 2 \alpha$ gene encoding the glucosidase II alpha subunit and engaged in protein glycosylation, which had a frame-shift mutation in RUT-C30 [17]. In addition, several mutations potentially affecting extracellular enzyme trafficking and secretion have also been identified; examples include genes encoding a plasma membrane-related protein (tre81136), a cell wall protein (tre124295), an ARP2/3 complex protein (tre2439), and actin-interacting protein 3 (tre35386) [18, 19]. Although recently reported followup work has attempted to explain how these mutations affect phenotype (as defined by the transcriptome and cultivation behavior) [20], direct experimental evidence for the actual ability of each of these targets to contribute to the final protein secretion is still lacking at the cellular level. Although the generation of knockout (KO) mutants for these genes might be a direct way to check whether gene functions contribute to hypersecretion, the construction of hundreds of $\mathrm{KO}$ mutants in $T$. reesei would be time consuming and difficult to complete. Given that Neurospora crassa has a close phylogenetic relationship with $T$. reesei and possesses a nearly complete set of genome-wide gene deletion mutants, thereby making it a powerful tool for use in genetic studies [21], we reasoned that comparative genomic screening of $N$. crassa mutants could be applied as an alternative approach to study functions of mutated genes in T. reesei RUTC- 30 .

In the present work, systematic screening of $86 \mathrm{~N}$. crassa $\mathrm{KO}$ mutants for mutated RUT-C30 orthologs was used to identify at least 12 genes with negative effects on lignocellulase secretion and 4 genes with positive effects. We further examined two genes that encode subunits of the adaptor protein 3 (AP-3) complex mediating hypersecretion in $N$. crassa and explored the possible conservation of the underlying mechanism in other ascomycetes including T. reesei. On the basis of our findings, we proposed a novel strategy to achieve the hypersecretion of lignocellulase in filamentous fungal systems by disrupting the natural balance between protein secretion and nutritionally required protein degradation.

\section{Results}

\section{Screening of mutants}

To address whether genes that bear mutations in $T$. reesei RUT-C30 are genuinely involved in protein secretion, we tested the secretion capacity of orthologous gene KO mutants in $N$. crassa. Le Crom et al. [19] previously identified 223 single nucleotide variants, 15 small insertions/deletions, and 18 larger deletions in RUT-C30, with an additional 17 mutations reported by Vitikainen et al. [18]. After excluding mutated genes shown to lack protein secretory functions in the two published studies, 164 mutated T. reesei genes were selected for ortholog calling in N. crassa using the local BLASTp program. We found 140 orthologs in $N$. crassa, among which 86 had homokaryotic gene $\mathrm{KO}$ mutants, including $\Delta$ cre 1 
(NCU08807) [22]. We further screened these 86 mutants by determining their lignocellulase secretion capacity through batch culturing with microcellulose $(2 \%[\mathrm{w} / \mathrm{v}]$ Avicel) as the carbon source and yeast extract $(0.75 \% \mathrm{w} / \mathrm{v})$ as the nitrogen source. Similar to T. reesei, lignocellulases accounted for most of the secretome (91\% by weight) in $N$. crassa, with the four major components $(\mathrm{CBH}-$ 1, $\mathrm{CBH}-2$, EG-1, and BG-2) representing about $65 \%$ of the total cellulase cocktail proteins [23, 24]. Measured concentrations of extracellular proteins, used to reflect lignocellulase secretion capacity, are shown in Fig. 1 and Table 1. We found lignocellulase production to be reduced by more than $25 \%$ in 4 mutants compared with the wild-type strain (WT), with secretions elevated by more than $25 \%$ in 12 strains. Similar to $\Delta$ cre 1,5 of these 12 mutants had markedly increased secretion of lignocellulases, including NCU07880 (annotated as a protein kinase), whose deletion increased the amount of secreted protein by approximately $35 \%$ compared with WT $N$. crassa and NCU01242 (encoding a protein predicted as a G2/mitotic-specific cyclin), whose deletion increased protein secretion by about $32 \%$. Both these genes are involved in cell cycle-related functions. In addition, deletion of NCU01161 (encoding a protein functionally similar to actin polymerization protein Bzz1 and associated with endo- or exocytic pathways) increased protein secretion by approximately 34\%. Loss of NCU07492, encoding a hypothetical protein, enhanced protein secretion by more than $30 \%$. Finally, loss in N. crassa of the NCU03998 gene, whose counterpart in T. reesei RUTC30, tre53811, has a mutation in its exon that changes serine $^{73}$ to leucine [18], increased secreted protein levels

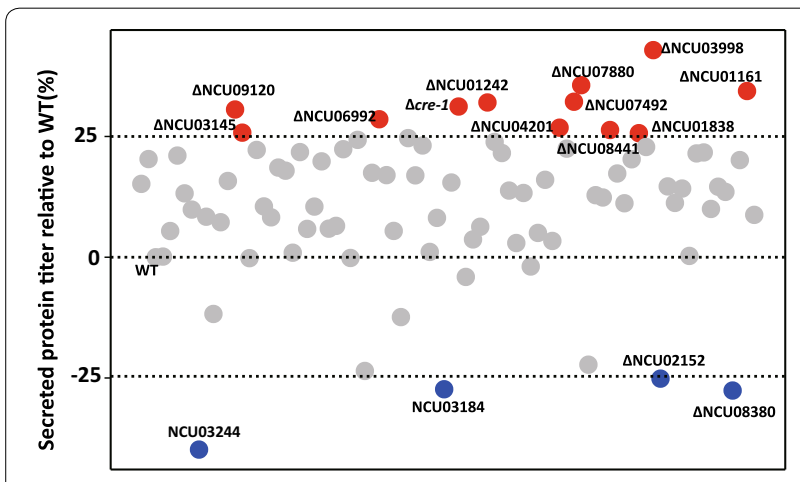

Gene knock-out mutants : hyper-secretion No change hypo-secretion

Fig. 1 Percentages of secreted protein in 86 Neurospora crassa knockout (KO) mutants relative to the wild type (WT). After inoculating conidia from each strain into Avicel medium and culturing for 7 days, the secreted protein titers were measured and are displayed on the scatter plot. Mutants with secretion capacities altered by more than 25\% compared with the WT are indicated as follows: red dots hypersecretion; blue dots hyposecretion. up to $42 \%$ compared with the WT; this mutant exhibited the highest protein secretion among tested strains. NCU03998 was predicted to encode the $\mu$ subunit of the AP-3 complex. Because the way in which the AP-3 complex affects lignocellulase secretion has not been previously reported, we designated the gene at locus NCU03998 as Ncap3m in this study and focused on its functional characterization.

\section{Ncap3m encodes the $\mu$ subunit of the AP-3 complex in N. crassa}

The AP-3 complex is well conserved in various eukaryotes $[25,26]$. We were able to identify NcAP3m sequence homologs in Saccharomyces cerevisiae (identity: 25\%, E value: $4 \times 10^{-15}$ ), Arabidopsis thaliana (identity: $32 \%$, $E$ value: $2 \times 10^{-27}$ ), Drosophila melanogaster (identity: $34 \%, E$ value: $9 \times 10^{-33}$ ), Mus musculus (identity: $29 \%, E$ value: $2 \times 10^{-32}$ ), and even Homo sapiens (identity: $29 \%$, $E$ value: $2 \times 10^{-32}$ ). To reveal the phylogenetic position of Ncap3m within eukaryotes, we generated the phylogenetic tree of AP-3 complex $\mu$-subunit proteins shown in Fig. 2. Proteins in this tree were significantly divided into three major groups corresponding to fungi, plants, and metazoans. Ncap3m was clustered along with its ortholog tre53811 from T. reesei (identity: $57 \%, E$ value: $0.00)$ in a well-supported clade within the fungal group.

\section{Ncap3m is involved in lignocellulase production in $N$. crassa}

To further determine how Ncap3m impacts lignocellulase production in N. crassa, we performed batch culturing of $\Delta N c a p 3 m$ using Avicel or xylan as the sole carbon source, with the WT used as the positive control. In batch cultures with $2 \%(\mathrm{w} / \mathrm{v})$ Avicel, the protein secretion titer of $\Delta N c a p 3 m$ increased by more than $80 \%$ compared with the WT. Filter paper activity (FPA) and xylanase activities were, respectively, 44 and $80 \%$ higher in the mutant than in the WT, with endoglucanase and exoglucanase activities showing respective increases of approximately 24 and $20 \%$ (Fig. 3a). When we changed the culture conditions to $2 \%$ xylan as the sole carbon source, thereby allowing xylanase to be specifically induced and secreted [27], the extracellular protein concentration of the $\Delta$ Ncap $3 m$ mutant was improved by approximately $50 \%$ and xylanase activity increased by about $100 \%$ (Fig. 3c). All of these results were additionally confirmed by sodium dodecyl sulfate-polyacrylamide gel electrophoresis (SDS-PAGE) profiling of the extracellular secretome (Fig. 3b, d), with known cellulase proteins from a previously reported LCMS analysis used as a reference [23].

To further show that the lignocellulase hypersecretion phenotype was caused by the loss of Ncap3m in $N$. crassa rather than other unknown genetic mutations, 
Table 1 List of extracellular proteins produced by Neurospora crassa mutants for genes orthologous to mutation-hit targets in Trichoderma reesei RUTC30

\begin{tabular}{|c|c|c|c|c|c|c|}
\hline No. & $\begin{array}{l}\text { Mutant } \\
\text { (FGSC) }\end{array}$ & $\begin{array}{l}\text { Orthologs in } \\
N . \text { crassa }^{\mathrm{a}}\end{array}$ & RUT C30 targets ${ }^{b}$ & Gene product names & $\begin{array}{l}\text { Protein conc. } \\
\text { (mg/L) }\end{array}$ & $\begin{array}{l}\text { Increased } \\
\text { vs WT }(\%)^{c}\end{array}$ \\
\hline 1 & 11701 & NCU03998 & jgi|Trire2|53811| & Adaptor protein complex 3 Mu3A & $821.38 \pm 21.83$ & 42.9 \\
\hline 2 & 17965 & NCU07880 & jgi|Trire2|124172| & Protein kinase & $780.01 \pm 32.87$ & 35.64 \\
\hline 3 & 19384 & NCU01161 & jgi|Trire2|68926| & Actin polymerization protein Bzz1 & $773.06 \pm 31.51$ & 34.44 \\
\hline 4 & 19393 & NCU07492 & jgi|Trire2|122689| & Hypothetical protein & $758.58 \pm 21.01$ & 32.21 \\
\hline 5 & 15743 & NCU01242 & jgi|Trire2|69437| & G2/mitotic-specific cyclin & $759.76 \pm 41.93$ & 32.06 \\
\hline 6 & 10372 & NCU08807 & jgi|Trire2|120117| & cre-1 & $755.26 \pm 51.79$ & 31.22 \\
\hline 7 & 11964 & NCU09120 & jgi|Trire2|76515| & $\begin{array}{l}\text { Lysine-specific histone demethylase } \\
\text { Aof2 }\end{array}$ & $750.7 \pm 20.09$ & 30.6 \\
\hline 8 & 15360 & NCU06992 & jgi|Trire2|31118| & DNA repair protein Nse1 & $737.31 \pm 37.42$ & 28.59 \\
\hline 9 & 16681 & NCU04201 & jgi|Trire2|106234| & Signal transduction protein Syg1 & $729.66 \pm 38.29$ & 26.84 \\
\hline 10 & 12039 & NCU08441 & jgi|Trire2|123786| & Non-ribosomal peptide synthetase & $729.27 \pm 112.17$ & 26.36 \\
\hline 11 & 12276 & NCU03145 & jgi|Trire2|78301| & Vacuolar membrane zinc transporter & $723.79 \pm 40.24$ & 25.81 \\
\hline 12 & 13177 & NCU01838 & jgi|Trire2|65106| & Nitrilase & $722.38 \pm 13.8$ & 25.71 \\
\hline 13 & 11468 & NCU04142 & jgi|Trire2|123114| & Heat shock protein 80 & $717.96 \pm 59.51$ & 24.68 \\
\hline 14 & 12062 & NCU07498 & jgi|Trire2|79304| & DNA excision repair protein Rad2 & $715.8 \pm 57.68$ & 24.32 \\
\hline 15 & 16319 & NCU02842 & jgi|Trire2|121087| & Hypothetical protein & $712.55 \pm 32.87$ & 23.89 \\
\hline 16 & 18830 & NCU03811 & jgi|Trire2|121453| & Hypothetical protein & $710.24 \pm 91.52$ & 23.16 \\
\hline 17 & 19831 & NCU03545 & jgi|Trire2|82153| & Hypothetical protein & $706.55 \pm 32.91$ & 22.85 \\
\hline 18 & 11956 & NCU08809 & jgi|Trire2|74765| & Hypothetical protein & $704.12 \pm 19.44$ & 22.5 \\
\hline 19 & 16594 & NCU03914 & jgi|Trire2|64375| & Glucan 1,3-beta-glucosidase & $704.88 \pm 64.43$ & 22.38 \\
\hline 20 & 20296 & NCU08364 & jgi|Trire2|78320| & Choline sulfatase & $702.59 \pm 19.06$ & 22.23 \\
\hline 21 & 11040 & NCU05411 & jgi|Trire2|70071| & Pathway-specific nitrogen regulator & $701.46 \pm 65.9$ & 21.78 \\
\hline 22 & 19539 & NCU07334 & jgi|Trire2|68425| & Uracil permease & $698.2 \pm 25.84$ & 21.72 \\
\hline 23 & 18971 & NCU00503 & jgi|Trire2|71037| & Nonselective cation channel protein & $700.38 \pm 66.13$ & 21.59 \\
\hline 24 & 13289 & NCU00427 & jgi|Trire2|80691| & Hypothetical protein & $698.5 \pm 19.9$ & 21.52 \\
\hline 25 & 15939 & NCU00754 & jgi|Trire2|58561| & Multidrug resistant protein & $697.71 \pm 79.14$ & 21.05 \\
\hline 26 & 13436 & NCU05089 & jgi|Trire2|64882| & MFS monocarboxylate transporter & $692.03 \pm 30.99$ & 20.33 \\
\hline 27 & 16570 & NCU04886 & jgi|Trire2|28409| & MFS multidrug transporter & $693.08 \pm 69.06$ & 20.3 \\
\hline 28 & 16331 & NCU03068 & jgi|Trire2|58790| & Glycerol-3-phosphate phosphatase 1 & $690.98 \pm 32.01$ & 20.14 \\
\hline 29 & 20407 & NCU11050 & jgi|Trire2|75105| & DUF455 domain-containing protein & $689.63 \pm 40.55$ & 19.86 \\
\hline 30 & 11084 & NCU09549 & jgi|Trire2|26255| & $\begin{array}{l}\text { C6 zinc finger domain-containing } \\
\text { protein }\end{array}$ & $683.56 \pm 79.47$ & 18.58 \\
\hline 31 & 18230 & NCU09887 & jgi|Trire2|67030| & Drp1p & $678.63 \pm 42.93$ & 17.93 \\
\hline 32 & 19296 & NCU07276 & jgi|Trire2|67732| & ABC bile acid transporter & $677.02 \pm 69.64$ & 17.5 \\
\hline 33 & 15452 & NCU06309 & jgi|Trire2|22841| & Hypothetical protein & $674.61 \pm 18.59$ & 17.36 \\
\hline 34 & 15930 & NCU00648 & jgi|Trire2|59952| & Choline transporter & $675.67 \pm 114.38$ & 17.02 \\
\hline 35 & 13757 & NCU01420 & jgi|Trire2|54157| & Hypothetical protein & $672.9 \pm 36.3$ & 16.97 \\
\hline 36 & 15627 & NCU04626 & jgi|Trire2|28731| & G-protein coupled receptor & $667.67 \pm 37.79$ & 16.05 \\
\hline 37 & 15627 & NCU04626 & jgi|Trire2|123806| & G-protein coupled receptor & $667.67 \pm 37.79$ & 16.05 \\
\hline 38 & 16748 & NCU00799 & jgi|Trire2|40758| & Homocysteine S-methyltransferase & $669.17 \pm 128.93$ & 15.8 \\
\hline 39 & 14141 & NCU08499 & jgi|Trire2|58161| & GTPase-activating protein GYP5 & $663.7 \pm 13.31$ & 15.49 \\
\hline 40 & 13568 & NCU04521 & jgi|Trire2|54511| & Hypothetical protein & $665.59 \pm 127.62$ & 15.19 \\
\hline 41 & 20195 & NCU06578 & jgi|Trire2|104161| & KapG & $662.02 \pm 104.79$ & 14.69 \\
\hline 42 & 19233 & NCU01997 & jgi|Trire2|74570| & $\mathrm{ABC}$ transporter & $662.83 \pm 141.67$ & 14.63 \\
\hline 43 & 12060 & NCU07381 & jgi|Trire2|3027| & $\begin{array}{l}\text { DNA cross-link repair protein pso2/ } \\
\text { snm1 }\end{array}$ & $659.15 \pm 93.95$ & 14.25 \\
\hline 44 & 18123 & NCU07703 & jgi|Trire2|55887| & Hypothetical protein & $655.25 \pm 49.89$ & 13.82 \\
\hline 45 & 13910 & NCU05195 & jgi|Trire2|75072| & Hypothetical protein & $653.76 \pm 52.14$ & 13.55 \\
\hline
\end{tabular}


Table 1 continued

\begin{tabular}{|c|c|c|c|c|c|c|}
\hline No. & $\begin{array}{l}\text { Mutant } \\
\text { (FGSC) }\end{array}$ & $\begin{array}{l}\text { Orthologs in } \\
\text { N. crassa } a^{\mathrm{a}}\end{array}$ & RUT C30 targets ${ }^{b}$ & Gene product names & $\begin{array}{l}\text { Protein conc. } \\
\text { (mg/L) }\end{array}$ & $\begin{array}{l}\text { Increased } \\
\text { vs WT }(\%)^{c}\end{array}$ \\
\hline 46 & 11279 & NCU03125 & jgi|Trire2|79405| & NIMA-interacting protein TinC & $651.64 \pm 26.12$ & 13.32 \\
\hline 47 & 12569 & NCU00321 & jgi|Trire2|67024| & Hypothetical protein & $652.72 \pm 75.61$ & 13.23 \\
\hline 48 & 19403 & NCU07564 & jgi|Trire2|78465| & Siderophore iron transporter mirC & $648.04 \pm 5.97$ & 12.87 \\
\hline 49 & 15566 & NCU01961 & jgi|Trire2|59147| & DNA lyase Apn2 & $647.69 \pm 69.51$ & 12.39 \\
\hline 50 & 19712 & NCU00497 & jgi|Trire2|58391| & Hypothetical protein & $643.14 \pm 129.41$ & 11.27 \\
\hline 51 & 11150 & NCU00278 & jgi|Trire2|73912| & Hypothetical protein & $643.22 \pm 144.06$ & 11.2 \\
\hline 52 & 11459 & NCU00340 & jgi|Trire2|36543| & Transcription factor steA & $637.2 \pm 73.32$ & 10.54 \\
\hline 53 & 13984 & NCU05837 & jgi|Trire2|65104| & $\begin{array}{l}\text { Vacuolar protein sorting-associated } \\
\text { protein } 13 a\end{array}$ & $635.14 \pm 16.93$ & 10.5 \\
\hline 54 & 19183 & NCU05477 & jgi|Trire2|102776| & Hypothetical protein & $632.43 \pm 18.1$ & 10.02 \\
\hline 55 & 11061 & NCU08443 & jgi|Trire2|77513| & Transcription factor ace 3 & $635.36 \pm 137.21$ & 9.87 \\
\hline 56 & 13086 & NCU00541 & jgi|Trire2|80592| & Hypothetical protein & $627.2 \pm 73.28$ & 8.8 \\
\hline 57 & 16803 & NCU04809 & jgi|Trire2|82037| & MFS phospholipid transporter & $630.47 \pm 248.43$ & 8.4 \\
\hline 58 & 15478 & NCU08642 & jgi|Trire2|78268| & $\begin{array}{l}\text { Cyclic nucleotide-binding domain- } \\
\text { containing protein }\end{array}$ & $624.52 \pm 83.42$ & 8.28 \\
\hline 59 & 15647 & NCU00025 & jgi|Trire2|82499| & Integral membrane protein & $623.56 \pm 73.21$ & 8.17 \\
\hline 60 & 16280 & NCU04108 & jgi|Trire2|105874| & Isoamyl alcohol oxidase & $616.8 \pm 25.92$ & 7.26 \\
\hline 61 & 15869 & NCU00335 & jgi|Trire2|5140| & Pre-mRNA-splicing factor cwc15 & $612.18 \pm 15.31$ & 6.51 \\
\hline 62 & 13160 & NCU01633 & jgi|Trire2|62380| & Hexose transporter HXT13 & $611.29 \pm 19.85$ & 6.33 \\
\hline 63 & 19733 & NCU06832 & jgi|Trire2|112231| & Kinesin & $609.8 \pm 45.91$ & 5.93 \\
\hline 64 & 12341 & NCU09864 & jgi|Trire2|56726| & $\begin{array}{l}\text { 2-Oxoisovalerate dehydrogenase } \\
\text { alpha subunit }\end{array}$ & $608.18 \pm 4.15$ & 5.88 \\
\hline 65 & 11030 & NCU07788 & jgi|Trire2|52368| & Fungal specific transcription factor & $606.86 \pm 36.13$ & 5.47 \\
\hline 66 & 12282 & NCU06647 & jgi|Trire2|5403| & Enoyl-CoA hydratase/isomerase & $605.74 \pm 7.76$ & 5.43 \\
\hline 67 & 12018 & NCU02751 & jgi|Trire2|120806| & Serine/threonine-protein kinase & $605.22 \pm 59.32$ & 5.05 \\
\hline 68 & 11677 & NCU01868 & jgi|Trire2|59388| & MFS maltose permease MalP & $595.74 \pm 3.43$ & 3.71 \\
\hline 69 & 18917 & NCU05459 & jgi|Trire2|65773| & Mitochondrial AAA ATPase & $595.8 \pm 64.3$ & 3.39 \\
\hline 70 & 19405 & NCU07574 & jgi|Trire2|22294| & Hypothetical protein & $591.48 \pm 5.41$ & 2.96 \\
\hline 71 & 17946 & NCU04755 & jgi|Trire2|45456| & $\begin{array}{l}\text { Protein kinase domain-containing } \\
\text { protein ppk32 }\end{array}$ & $581.14 \pm 12.76$ & 1.12 \\
\hline 72 & 17081 & NCU01044 & jgi|Trire2|63464| & Hypothetical protein & $580.08 \pm 10.41$ & 0.95 \\
\hline 73 & 19059 & NCU08307 & jgi|Trire2|56077| & Hypothetical protein & $579.21 \pm 100.55$ & 0.3 \\
\hline 74 & 17389 & NCU02337 & jgi|Trire2|80332| & Mitochondrial carrier protein & $576.12 \pm 32.03$ & 0.14 \\
\hline 75 & 2489 & \#\#\#\#\# & \#\#\#\#\# - n & Wild type & $575.45 \pm 36.81$ & 0 \\
\hline 76 & 16836 & NCU04847 & jgi|Trire2|52520| & cyclin & $573.97 \pm 15.22$ & -0.13 \\
\hline 77 & 20073 & NCU06341 & jgi|Trire2|44956| & MFS transporter & $573.58 \pm 9.57$ & -0.17 \\
\hline 78 & 19245 & NCU02220 & jgi|Trire2|64866| & hypothetical protein & $563.98 \pm 19.59$ & -1.9 \\
\hline 79 & 12078 & NCU00523 & jgi|Trire2|50268| & NAD-dependent deacetylase sirtuin-2 & $551.74 \pm 23.25$ & -4.05 \\
\hline 80 & 12072 & NCU04203 & jgi|Trire2|121351| & Glucosidase II alpha subunit & $508.03 \pm 35.04$ & -11.73 \\
\hline 81 & 13475 & NCU07119 & jgi|Trire2|60458| & Nonribosomal peptide synthase 2 & $503.65 \pm 12.98$ & -12.37 \\
\hline 82 & 19165 & NCU05213 & jgi|Trire2|75074| & Hypothetical protein & $449.4 \pm 86.39$ & -22.22 \\
\hline 83 & 18185 & NCU08452 & jgi|Trire2|110570| & Hypothetical protein & $439.95 \pm 23.92$ & -23.52 \\
\hline 84 & 16956 & NCU02152 & jgi|Trire2|3400| & RRM domain-containing protein & $430.62 \pm 18.69$ & -25.11 \\
\hline 85 & 11357 & NCU03184 & jgi|Trire2|4921| & $\begin{array}{l}\mathrm{C} 2 \mathrm{H} 2 \text { conidiation transcription factor } \\
\mathrm{FlbC}\end{array}$ & $419.08 \pm 52.77$ & -27.31 \\
\hline 86 & 20306 & NCU08380 & jgi|Trire2|122050| & Plasma membrane phosphatase & $416.5 \pm 18.07$ & -27.57 \\
\hline 87 & 11360 & NCU03244 & jgi|Trire2|62053| & WD repeat protein & $346.47 \pm 27.22$ & -39.81 \\
\hline
\end{tabular}

a N. crassa orthologs: locus selected according to the N. crassa database (v7) (https://www.broadinstitute.org/annotation/genome/neurospora/MultiHome.html).

b RUTC30 targets: locus selected according to the T. reesei genome sequence web site (http://genome.jgi-psf.org/Trire2/Trire2.home.html).

c The increased percentage of secreted protein relative to the WT. Strains more than $25 \%$ increased or decreased compared with the WT are shown in italics. 


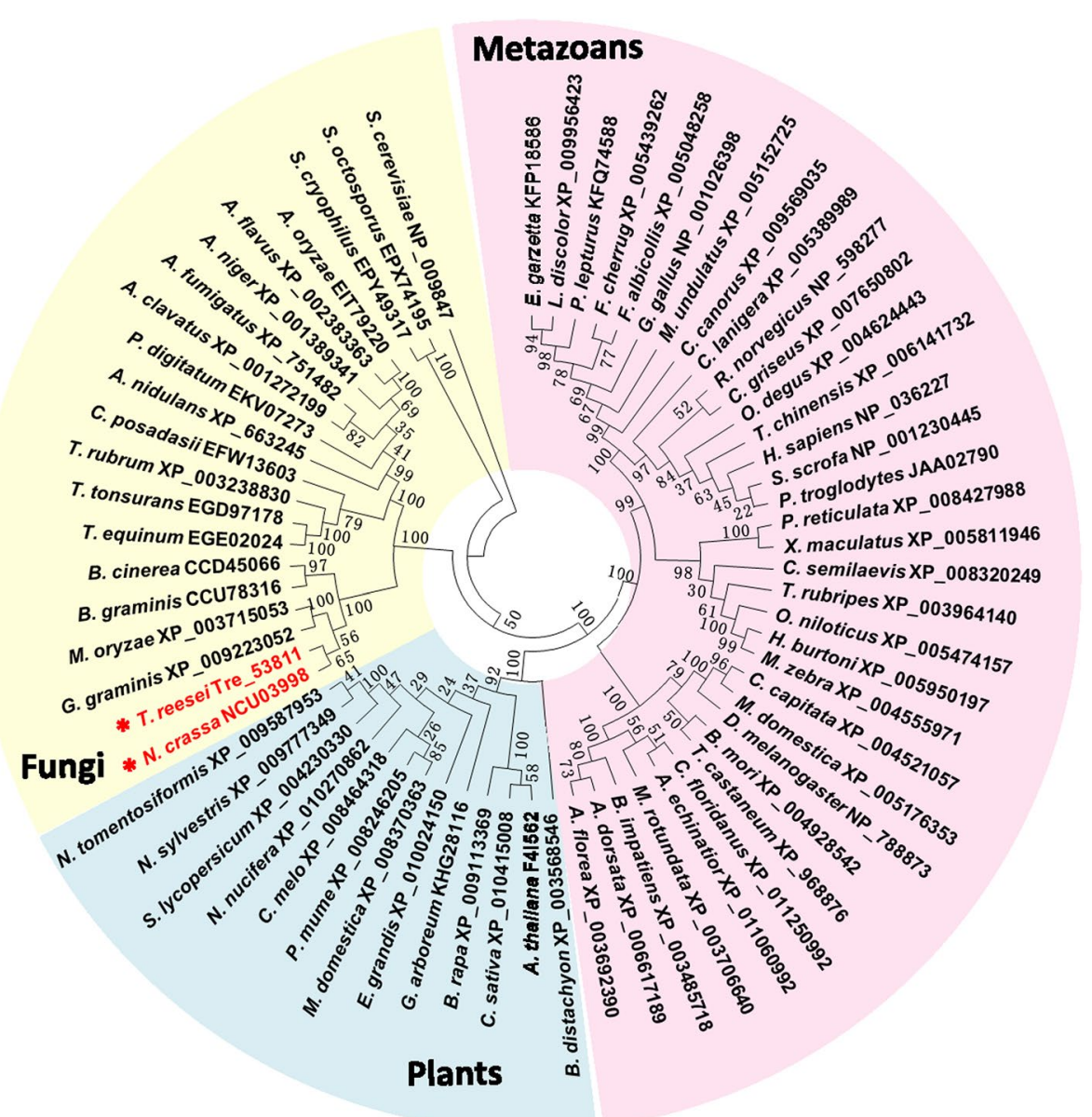

Fig. 2 Phylogenetic tree of adaptor protein 3 (AP-3) complex $\mu$-subunit proteins. Amino acid sequences were obtained from the NCBI database based on ortholog calling using the local BLASTp program. The phylogenetic analysis was performed by MEGA6 using the maximum likelihood method with 1,000 bootstrap replicates.

additional gene complementation assays were performed. We separately introduced enhanced green fluorescent protein (EGFP)-labeled Ncap3m under the control of ccg-1 [28] or native promoters into $\Delta N c a p 3 m$. Batch culturing under either Avicel or xylan utilization conditions revealed that both complemented isolates restored the altered secretion phenotype to levels similar to that of the WT. These results were verified by enzyme activity measurements (Fig. 4).

To investigate whether the hypersecretion observed in $N$. crassa due to the loss of the ap $3 m$ gene is conserved in T. reese $i$, we conducted an inter-complementation assay by introducing the ap $3 m$ gene of $T$. reesei into $\Delta N c a p 3 m$. We first cloned the 1,608-bp open reading frame (ORF) of Trap3m (tre53811) in the T. reesei QM6a cDNA (inhouse annotated; see "Methods"; Additional file 1: Figure S1, Additional file 2: Figure S2 for details) and placed it under the control of the $N$. crassa $c c g-1$ promoter. The plasmid was then transformed into $\Delta$ Ncap $3 m: h i s 3^{-} N$. crassa to form the strain Pc-Trap3m. When compared with its parental strain $\Delta$ Ncap3m:his3 ${ }^{-}$, protein secretion and enzyme activities in Pc-Trap3m were restored to WT levels, similar to the complemented strain Pc-ap3m (Fig. 4). This result implies that Trap $3 m$ can genetically complement the ap3m deletion phenotype in N. crassa. Taken together, these results indicate that the function of ap3m is evolutionarily conserved between the two lignocellulolytic ascomycetes.

Sub-cellular localization of the NcAP3m protein in N. crassa To assess NcAP3m protein sub-cellular localization, we observed the EGFP signal of NcAP3m-EGFP recombinant protein in young hyphae of the complemented strain $\Delta$ Ncap3m::Ncap3m-EGFP (Fig. 5). After pregrowth in minimal medium for $16 \mathrm{~h}$ followed by culturing in Avicel (2\% w/v) medium for another $4 \mathrm{~h}$, we found that NcAP3m-EGFP proteins were unevenly distributed in cytosol; they were primarily located in the extreme 

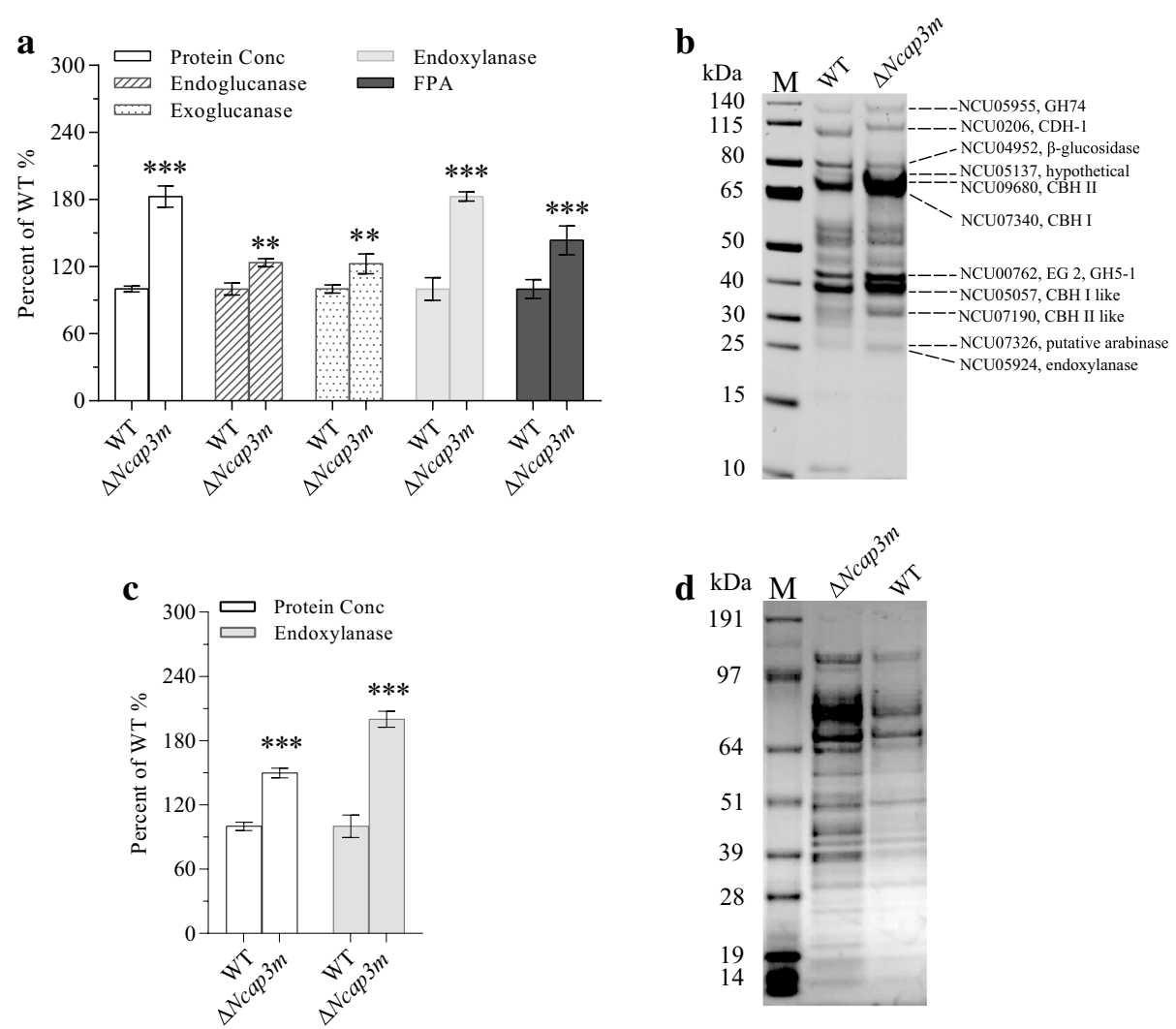

Fig. 3 Hypersecretion of lignocellulases by Neurospora crassa due to deletion of Ncap3m. Typical secretomes of the wild type (WT) and $\Delta$ Ncap3m are shown on a sodium dodecyl sulfate-polyacrylamide gel electrophoresis (SDS-PAGE) gel (a, c) after 7 days of culturing in Avicel (a, b) and xylan (c) d) media. Total extracellular protein concentration and enzyme activity (b, d) were measured and evaluated after normalization to the WT control according to percentage (standard error of the mean, $n=3$ ). Asterisks indicate significant differences from the WT $\left({ }^{* *} P<0.01 ;{ }^{* * *} P<0.001\right.$ ) based on one-way analysis of variance.
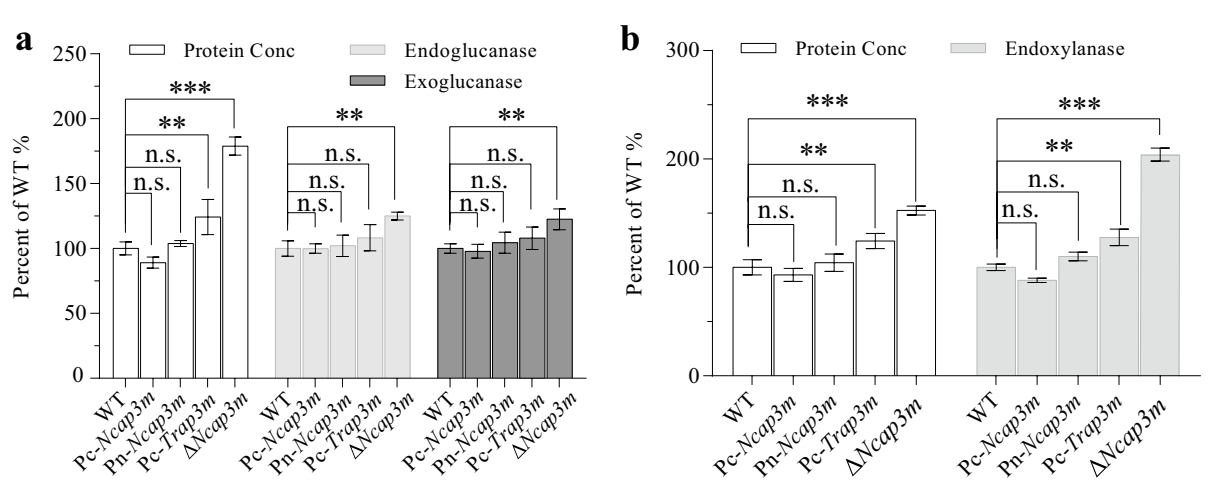

Fig. 4 Restoration of the $\Delta$ Ncap3m hypersecretion phenotype to normal wild-type (WT) levels by Ncap3m or Trap3m. The following strains were grown in $2 \%(\mathrm{w} / \mathrm{v})$ Avicel (a) or xylan $(\mathbf{b})$ media: the WT, the Ncap3m gene knockout $(K O)$ mutant $(\Delta N c a p 3 m)$, the Ncap3m-complemented strain under either the control of the ccg-1 strong promoter (Pc-Ncap3m) or the native promoter (Pn-Ncap3m), and a Trap3m-complemented strain introduced into $\Delta$ Ncap3m under the control of the $c c g-1$ strong promoter (Pc-Trap3m). After 7 days of culturing, the total extracellular protein concentration and endoglucanase (a) or endoxylanase (b) activity were measured. Data were normalized to the WT control according to percentage (standard error of the mean, $n=3$ ); asterisks indicate significant differences from the WT ${ }^{* *} P<0.01$; ${ }^{* *} P<0.001$; ns not significant) based on one-way analysis of variance. 
tip region of young hyphae, where they accumulated in a ring-like structure. Co-staining with membrane dye FM4-64 showed that NcAP3m-EGFP proteins overlapped with a structure known as the Spitzenkörper that has been reported to serve as a vesicle trafficking center in filamentous fungal cells [29]. This overlap implies that NcAP3m may be located in small secretory vesicles. When the culture time was extended to $48 \mathrm{~h}$, we found that NcAP3m-EGFP proteins no longer accumulated in tip regions. Instead, they were spotted around large vacuole-like structures near the tip region. This observed location is consistent with previous reports showing that the AP-3 complex in higher eukaryotes is primarily located in vacuoles and lysosomes and plays an important role in protein sorting and trafficking between the trans-Golgi network and vacuoles/lysosomes or endosomes [30, 31].

NCU06569 encoding the $\beta$ subunit of the AP-3 complex can also affect protein secretion in N. crassa

Whether other subunits of the AP-3 complex besides NcAP3m can affect protein secretion remain to be explored. Based on genome annotation, three other subunits of the AP-3 complex were found to exist in $N$. crassa; these were separately encoded by NCU06569 $(\beta$ subunit, Ncap3b), NCU04652 ( $\delta$ subunit, Ncap3 $\delta$ ) and NCU09461 ( $\sigma$ subunit, Ncap3 $\sigma$ ). Among the three genes, the KO mutant was available for Ncap3b (FGSC\#11856, $\Delta N c a p 3 b)$ and we therefore investigated its lignocellulase secretion capacity (Fig. 6a, b). When grown on $2 \%(\mathrm{w} / \mathrm{v})$ Avicel, the extracellular protein secreted by the $\Delta N c a p 3 b$ mutant was about $63 \%$ higher than that in the WT; FPA increased by approximately 37\%, while endoglucanase, exoglucanase, and xylanase activities increased by about $20 \%$ compared with the WT. These results demonstrate that $N c a p 3 b$ also markedly affected protein secretion. We next constructed a double mutant of two subunit-encoding genes of the AP-3 complex $(\Delta N$ cap $3 m \Delta N c a p 3 b)$. This double mutant had an extracellular protein secretion titer and related enzyme activities similar to those of the $\Delta N c a p 3 m$ single mutant (Fig. 6a, b), suggesting that NcAP3m is the critical subunit for full functionality of the AP-3 complex. Taking all these results into consideration, we deduced that the AP-3 complex has a significant impact on the secretion of proteins such as lignocellulases.

\section{Switch to Avicel for $4 \mathrm{~h}$ Switch to Avicel for $48 \mathrm{~h}$}
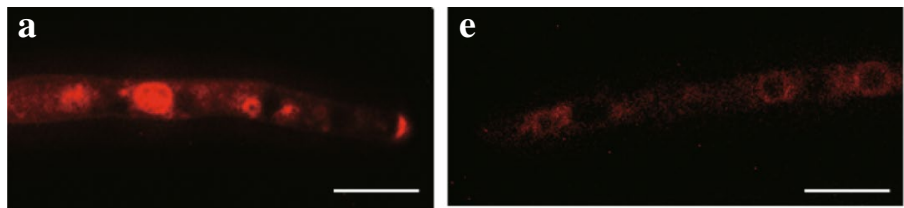

FM4-64
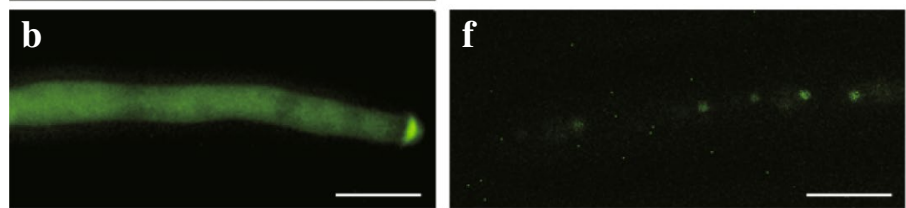

NcAP3m-EGFP
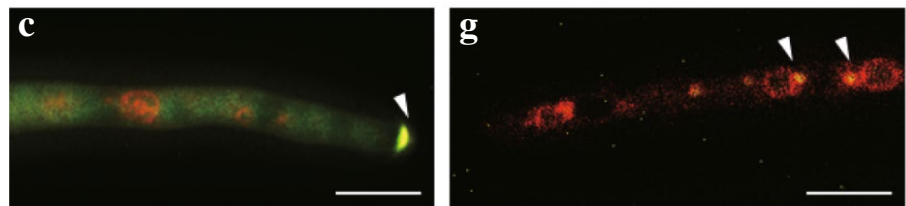

Merge
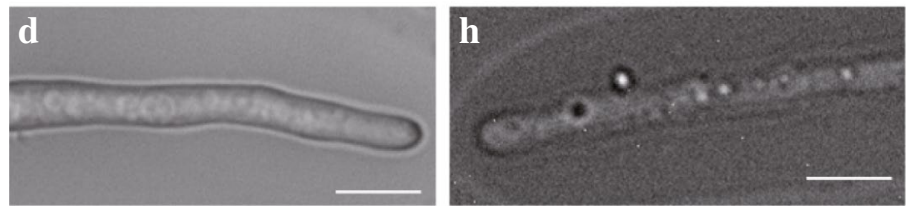

BF

Fig. 5 Sub-cellular localization of the adaptor protein 3 (AP-3) complex in Neurospora crassa. Wild-type (WT) and $\Delta$ Ncap3m strains were pre-grown in minimal medium with $2 \%(\mathrm{w} / \mathrm{v})$ sucrose as the sole carbon source for $16 \mathrm{~h}$ and then switched to Avicel medium to elicit lignocellulase production for another $4 \mathrm{~h}$ (initial stage; $\mathbf{a}-\mathbf{d}$ ) or $48 \mathrm{~h}$ (logarithmic stage; $\mathbf{e}-\mathbf{h}$ ). Extreme tip regions of mycelia of the WT and $\Delta$ Ncap $3 m$ were stained with $5 \mathrm{\mu g} / \mathrm{mL}$ of the membrane dye FM4-64 for $30 \mathrm{~min}$ to label membrane structures such as the Spitzenkörper or vacuoles (a, e). Locations of NcAP3m proteins were monitored by recording enhanced green fluorescent protein (EGFP) signal (b, f). Merged yellow fluorescence signal from FM4-64 and $\operatorname{EGFP}(\mathbf{c}, \mathbf{g})$ are denoted by white arrows in the photos. Each scale bar represents $10 \mu \mathrm{m}$. 
Lignocellulase gene transcriptional abundance is maintained at relatively higher levels during the late fermentation phase in AP-3 complex mutants

To further elucidate the hypersecretion phenotype of the AP-3 complex KO mutants, especially those of the key subunit NcAP3m, we monitored changes in the expressions of lignocellulase genes by quantitative real-time PCR (qPCR) during batch culturing of $\triangle N$ cap $3 m$. To avoid potential differences induced by variable growth rates of isolates, shift cultures were used. All strains were pre-grown in $2 \%(\mathrm{w} / \mathrm{v})$ sucrose until the formation of young hyphae and then switched to Vogel's medium containing $2 \%(\mathrm{w} / \mathrm{v})$ Avicel to induce lignocellulase gene expression. We monitored the expression patterns of three genes, namely, cbh-1 (NCU07340), cbh-2 (NCU09680), and gh5-1 (NCU00762), which respectively encode the major exoglucanase, cellobiohydrolase, and endoglucanase proteins in $N$. crassa $[23,24]$ (Fig. 7). In WT, all three genes had their highest expression values $4 \mathrm{~h}$ after induction; expression then rapidly decreased, normally within $48 \mathrm{~h}$, as culturing continued [32]. This phenomenon is known as repressed expression of secreted sequences (RESS), a feedback mechanism that selectively down-regulates transcription of genes encoding extracellular enzymes upon increased protein flux, thereby helping to reduce ER load [33-35]. In
$\Delta N c a p 3 m$, however, lignocellulase gene expression levels had not obviously decreased after $48 \mathrm{~h}$ and remained at a relatively high level compared with the WT until day 7 (Fig. 7). All of these results indicate that the RESS mechanism might be compromised in $\Delta N$ cap $3 m$, even though we still cannot interpret the detailed mechanism at present. This consistently higher induction might be an additional reason for the higher secretion of lignocellulase proteins in AP-3 complex defect mutants.

\section{Alkaline phosphatase influences lignocellulase secretion}

In $S$. cerevisiae, the AP-3 complex has been shown to be specifically involved in the transport of alkaline phosphatase (ALP) from the Golgi apparatus to vacuoles/ lysosomes [30]. Whether ALP affects lignocellulase secretion remains unclear. To address this question, we used the protein sequence of Pho8p, the sole vacuolar ALP in $S$. cerevisiae, as a query in BLASTp searches against the NCBI database $\left(E\right.$ value $\left.<1 \times 10^{-5}\right)$, thereby identifying two homologs in the $N$. crassa genome: NCU08997 (identity: $51 \%, E$ value: $1 \times 10^{-135}$ ) and NCU01376 (identity: $26 \%, E$ value: $2 \times 10^{-17}$ ). Further phylogenetic analysis revealed that the two ALP proteins were also well conserved in other filamentous fungal genomes, including that of T. reesei (Additional file 3: Figure S4). When we monitored the lignocellulase secretion capacity of gene $\mathbf{a}$

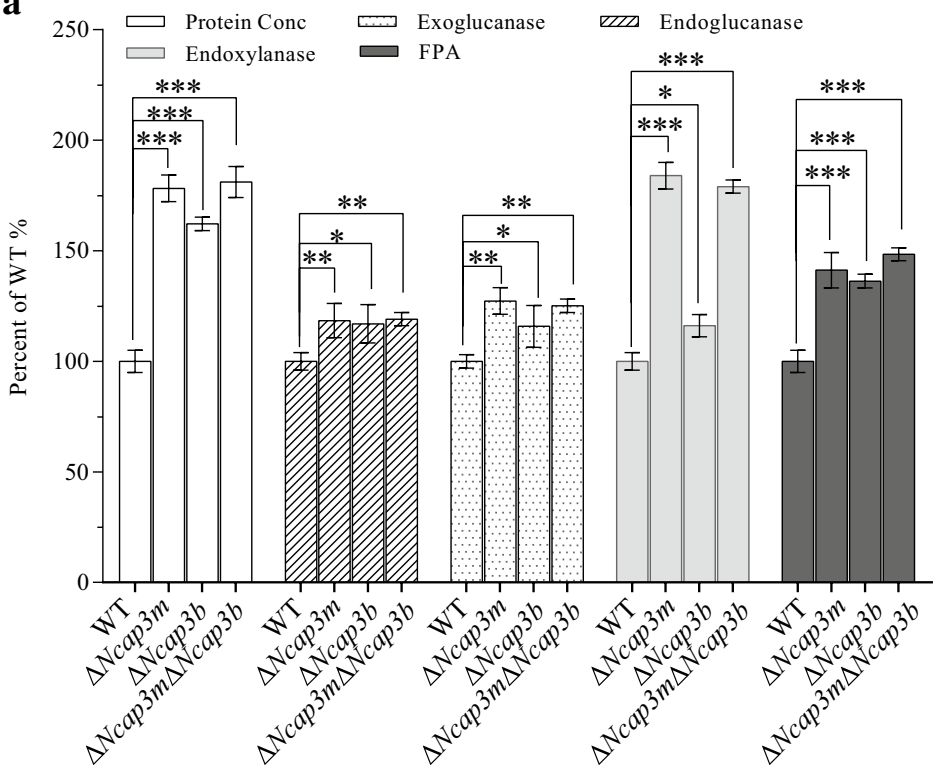

b $\mathrm{kDa}$

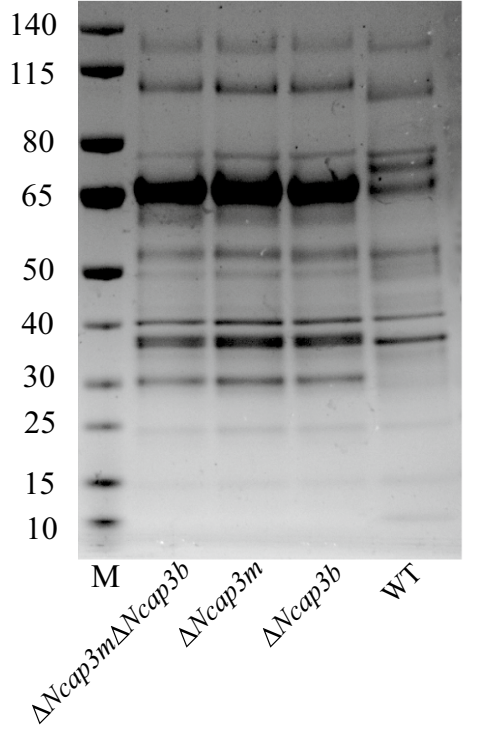

Fig. 6 Involvement of the adaptor protein 3 (AP-3) complex in lignocellulase secretion in Neurospora crassa. Conidia from the wild type (WT) and Ncap3m and Ncap3b single $(\Delta N c a p 3 m, \Delta N c a p 3 b)$ and double mutants $(\Delta N c a p 3 m \Delta N c a p 3 b)$ were separately inoculated into Avicel medium and batch cultured for 7 days. The typical secretome of each strain was then observed by sodium dodecyl sulfate-polyacrylamide gel electrophoresis (SDS-PAGE) (b), while total extracellular protein concentration and enzyme activity (a) were measured and displayed after normalization to the WT control according to percentage (standard error of the mean, $n=3$ ). Asterisks indicate significant differences from the WT $\left({ }^{*} P<0.05 ;{ }^{* *} P<0.01\right.$; $\left.{ }^{* * *} P<0.001\right)$ based on one-way analysis of variance. 


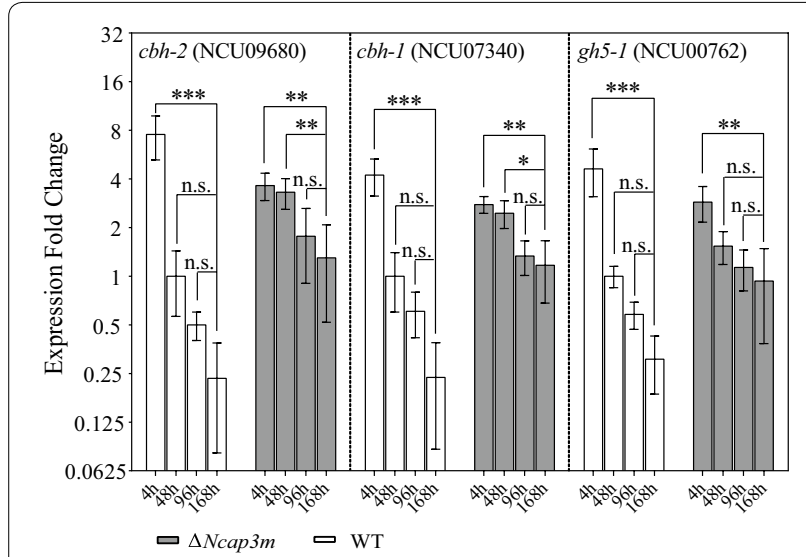

Fig. 7 Maintenance of high lignocellulase gene expression levels in $\Delta$ Ncap3m relative to the wild-type strain (WT) at the late fermentation stage. After $\Delta$ Ncap3m and WT conidia were grown on Avicel for $4,48,96$, or $168 \mathrm{~h}$, the transcriptional abundance of three major lignocellulase genes was evaluated by quantitative real-time PCR (qPCR). The data are normalized to the expression of the WT strain at $48 \mathrm{~h}$ for each tested gene, with actin (NCU04173) gene expression levels used as an endogenous control in all samples (standard error of the mean, $n=3$ ). Asterisks indicate significant differences from the control $\left({ }^{*} P<0.05 ;{ }^{* *} P<0.01 ;{ }^{* * *} P<0.001\right.$; $n$ s not significant) based on one-way analysis of variance.

KO mutants for the two targets using batch culturing, we discovered that the deletion of NCU08997 had a markedly positive effect on lignocellulase secretion and the deletion of NCU01376 yielded no obvious phenotypes (Fig. 8). This result suggests that NCU08997 serves as the main functional vacuolar ALP in N. crassa. Our findings also indicate that ALP involved in efficient protein degradation may be responsible for the observed induction of lignocellulase hypersecretion caused by the AP-3 complex malfunction.

\section{Discussion}

Because lignocellulases are a pivotal factor in the biorefinery process, improvement of their production titers could effectively reduce the cost of biofuel production. Most currently used industrial hosts, such as $T$. reesei (Hypocrea jecorina) and Aspergillus spp., can achieve an outstanding output; because most of these strains were generated by random mutagenesis, however, a clear genetic basis underpinning the requirements of effective secretion has not yet been elucidated [11]. Previous work comparing the genomic data of two $T$. reesei hyperproducing mutants, NG14 and RUT-C30, with their reference isolate QM6a, uncovered more than 200 mutagenic including many single-nucleotide substitutions, some small insertions/deletions, and more than $100 \mathrm{~kb}$ of larger genomic DNA deletions [19]. Some of these mutagenic events have affected genes engaged in functions such as secretion/vacuolar targeting, mRNA stability, and transcription, and thus might contribute to hypersecretion phenotypes. Meanwhile, independent work using high-resolution array comparative genomic hybridization analysis of T. reesei NG14 and RUT-C30 uncovered an additional 17 previously undocumented mutation sites. Importantly, two deletions identified in RUT-C30, of a large $85-\mathrm{kb}$ genomic DNA segment and a transcription factor, were both determined to not be involved in cellulase production [18]; this raises the question of whether other mutagenic events have potential roles in boosting secretion capacity. A large-scale screening of each mutation in the $T$. reesei system seems unreachable; however, $N$. crassa is genetically very similar to T. reesei and possesses a nearly complete genomic $\mathrm{KO}$ strain set, which provides an alternative opportunity to test the possible contribution of chromosomal mutations to effective secretion. In this work, we systematically screened deletion mutants of $86 \mathrm{~N}$. crassa genes that were orthologs of T. reesei genes having mutations in RUT-C30 relative to its parental strain QM6a. Further examination of the lignocellulase production capacities of these corresponding KO strains by batch culturing revealed 12 strains, including well-known mutant $\Delta$ cre-1 [16], which were able to promote protein secretion by more than $25 \%$ in comparison with the WT. Among these strains, we found several targets involved in cell cycle-related functions, such as NCU07880, the homolog of the Aspergillus nidulans Cdc2-related kinase gene $n p k A$ [36]; NCU01242, which encodes G2/mitotic-specific cyclin; and NCU06992,

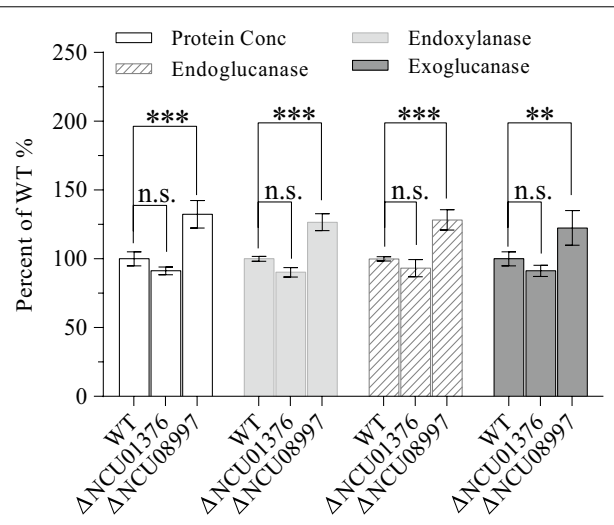

Fig. 8 Screenings of two putative alkaline phosphatase knockout (KO) mutants in Neurospora crassa to reveal their potential functional association with lignocellulase secretion. Conidia of $\Delta$ NCU08997, $\Delta$ NCU01376, and wild-type (WT) strains were separately inoculated into Avicel medium and batch cultured for 7 days; total extracellular protein concentration and enzyme activity were then measured and normalized to the WT control according to percentage (standard error of the mean, $n=3$ ). Asterisks indicate significant differences from the control ( ${ }^{* *} P<0.01$; ${ }^{* * *} P<0.001$; ns not significant) based on one-way analysis of variance. 
which encodes a homolog of the fission yeast DNA repair protein Nse1 possibly contributing to genome stability [37]. Previous work on T. reesei has demonstrated that effective secretion is related to low growth rate [38], implying that interruption of the cell cycle may contribute to enhanced lignocellulase secretion. Moreover, NCU01161 encodes an actin polymerization protein that acts as the homolog of S. cerevisiae BZZ1 [39]. In filamentous fungi, actin cytoskeleton organization is commonly linked with endo- or exocytic pathways [40]; thus, hypersecretion induced by a defect in NCU01161 might result from alteration of such pathways. In addition, our screening identified several deletion strains, namely, $\Delta$ NCU02152, $\triangle$ NCU03184, $\Delta$ NCU08380, and $\triangle \mathrm{NCU} 03244$, with decreased secretion capacities, which suggests that they play positive roles in regulating lignocellulase secretion. All four strains were found to have secretion that was decreased by more than $25 \%$ compared with WT $N$. crassa, although the detailed mechanisms remain to be elucidated by further studies. Unlike previous observations, we found that deletion of NCU04203, which encodes the alpha subunit of glucosidase II, decreased lignocellulase production by approximately $10 \%$ rather than promoting its secretion [17]. We speculate that improperly glycosylated enzyme protein might be degraded intracellularly rather than being secreted to the extracellular matrix. Despite the identification of dozens of mutations in many interesting genes associated with protein synthesis and secretion, it should be noted that our screenings found that most are likely non-functional mutations-at least, no obvious phenotype could be seen at single-gene disruption levels. These results clearly agree with the idea that the hypersecretion phenotype of $T$. reesei RUT C30 is not caused by any single-gene mutation. Because only single-gene $\mathrm{KO}$ stocks were screened in this study, the interactions among these mutant alleles could not be elucidated by the present work. However, $N$. crassa can be easily sexually crossed to generate double-, triple-, and multiple-gene mutants, which would help to further investigations of the mechanism of hypercellulase production in RUT C30 in future.

Notably, we found that deletion of Ncap3m (NCU03998) in N. crassa achieved the highest extracellular protein yield among all screened mutants, with amounts of secreted proteins comparable to $\Delta$ cre-1. Furthermore, the results of FPA and xylanase activity measurements confirm the observed enhancement of cellulase and hemicellulase production in this strain, suggesting that NCU03998 functions in the regulation of secretion without enzyme specificity. Protein sequence alignment revealed that NCU03998 encodes a protein homologous to the AP-3 complex subunit $\mu 3 \mathrm{a}$ in mammals and Amp3p in S. cerevisiae. Adaptor protein complexes
(AP complexes) mediate the formation of vesicles and participate in intra-organelle membrane trafficking in eukaryotes [25, 41, 42]. To date, five AP complexes (AP-1 to AP-5) have been identified [43], and AP-3 has been extensively studied in mammals, flies, Arabidopsis, and yeast $[25,41,42,44]$. In mammalian cells, the AP-3 complex is a four-subunit heterotetrameric complex consisting of two large subunits ( $\beta 3 \mathrm{a}$ and $\delta$ ), a medium subunit $(\mu 3 \mathrm{a})$ and a small subunit $(\sigma 3)[45,46]$. In addition to Ncap3m, we also tested the secretion capacity of another AP-3 complex large-subunit- $\beta 3$ a mutant ( $\triangle$ NCU06569, $\Delta N c a p 3 b)$ in $N$. crassa; this deletion mutant also displayed an enhanced secretion phenotype similar to that of $\Delta N c a p 3 m$, suggesting that the AP-3 complex is indeed involved in lignocellulase secretion. Moreover, we successfully complemented Trap $3 m$ in $\Delta N$ cap $3 m$, implying that the AP-3 complex, and Trap3m in particular, may contribute to the hypersecretion phenotype of the T. reesei RUT-C30 strain.

Previous work on $S$. cerevisiae has demonstrated that the AP-3 complex is required for the intracellular retention of the major chitin synthase Chs3p located in the plasma membrane [47], suggesting that the AP-3 complex can influence secretory protein secretion. Details of the process remain to be elucidated, however, especially in filamentous fungal systems. In addition, the S. cerevisiae AP-3 complex has been shown to be specifically involved in the transport of ALP from the Golgi apparatus to vacuoles/lysosomes. Deletion of Amp3p resulted in a pronounced accumulation of ALP in cytoplasm rather than at the vacuolar membrane [30], suggesting that Amp3p plays an important role in ALP location. PHO8 encodes the sole vacuolar integral membrane ALP in $S$. cerevisiae [48] and is commonly used as a marker protein for monitoring autophagy $[49,50]$. ALP can efficiently dephosphorylate a variety of phosphopeptides, thereby releasing phosphate groups [51]. Although the exact physiological function of ALP remains unclear, research using animal models has demonstrated that phosphate can mediate autophagy stimulation [52], which has been partially interpreted to be the potential link between ALP and autophagy. Notably, a previous study of fibroblasts, which are secretory cells that can secrete large quantities of collagen for immunity functions, found that ALP activity can contribute to collagen degradation [53]. That observation raises the possibility that ALP may also contribute to the degradation of other secretory proteins such as lignocellulases in filamentous fungi. In the present study, we found that NcAP3m protein following brief exposure ( $4 \mathrm{~h}$ ) of $N$. crassa cells to microcellulose was not located at vacuole/lysosome-like structures as previously reported in eukaryotes. Instead, NcAP3m was located in the tip region of fungal hyphae and likely overlapped with 
the Spitzenkörper comprising numerous small secretory vesicles. This localization implies that NcAP3m is a critical component of the secretory vesicle membrane and mediates extracellular enzyme secretion. As the culture time was increased to $48 \mathrm{~h}$, rapid lignocellulase synthesis was observed, protein secretion flux increased exponentially, and NcAP3m protein was found to have relocated to large vacuole/lysosome-like structures. As vacuole/lysosome function is usually linked to protein degradation and nitrogen source recycling, we speculate that high rates of secretory protein synthesis and secretion should cause fungal cells to temporarily experience nitrogen source starvation; this in turn would activate the recycling of nutrients via partial lignocellulase degradation. The AP-3 complex consequently seems to balance the secretion and degradation of secretory proteins in fungal cells. Malfunction of the AP-3 complex attenuates the degradation process, which in turn enhances secretion. To check whether ALP contributes to lignocellulase degradation, we also tested lignocellulase secretion capacity in two putative ALP gene KO mutants and found that the deletion of the ALP encoded by NCU08997 led to a hypersecretion phenotype in N. crassa. Because the level of hypersecretion was lower than that expected based on the disruption of the AP-3 complex, we hypothesize that other classes of vacuolar enzymes involved in the degradation process may also require the AP- 3 complex for correct sorting. Nevertheless, the means by which the AP-3 complex mediates these degradation enzymes locations in N. crassa remains unknown and requires further investigation. We found that the deletion of NCU03145, which encodes a vacuolar membrane zinc transporter in $N$. crassa, also enhances lignocellulase secretion. In $S$. cerevisiae, the counterpart of NCU03145 is ZRC1, which functions in transporting zinc from cytosol to vacuole for storage [54]. Vacuolar zinc transporters have been shown to contribute to the maintenance of ALP accumulation and activity [55], implying that the hypersecretion phenotype of $\triangle \mathrm{NCU} 03145$ might also result from disruption of ALP function. Considering all of these results, we propose a novel model, illustrated in Fig. 9, to explain how the AP-3 complex determines the ultimate fate of lignocellulase.

According to our results, the expression of lignocellulase genes at the transcriptional level had an effect in $\Delta N c a p 3 m$ and $\Delta N c a p 3 b$. The typical RESS phenomenon attenuated in these mutants. Although we could not determine the precise mechanism, we speculate that this change may be an indirect consequence of lignocellulase secretion induced by AP-3 complex malfunction, which could yield more efficient degradation of cellulose to produce biopolymer inducers to maintain lignocellulase gene expression. Although the xylanase activity change was proportionate to the protein enhancement level, we additionally noticed that the activity increase of cellulases such as endoglucanase under Avicel conditions, approximately $20 \%$, was not as high as the enhancement of secreted enzymes protein titers $(80 \%)$. We currently have no good explanation for this observation. As mentioned above, deletion of S. cerevisiae amp3 resulted in a pronounced accumulation of ALP in the cytoplasm, raising the possibility that ALPs in $\Delta N c a p 3 m$ or $\Delta N c a p 3 b$ may be secreted in the extracellular matrix, thus modifying lignocellulases and interfering with the enzyme activity.

\section{Conclusions}

In this study, we used gene deletion mutant stocks of $N$. crassa to investigate the function of orthologs of T. reese $i$ genes whose mutations might contribute to the hypersecretion phenotype of the RUT-C30 strain. We identified several potential targets, especially the AP-3 complex, involved in the lignocellulase secretion pathway. This study provides a novel view of lignocellulase secretion and suggests a new strategy for future improvement of fungal strains.

\section{Methods}

\section{Strains}

Neurospora crassa strains, including the WT (FGSC\#2489) as well as gene KO mutants, were obtained from the Fungal Genetics Stock Center (FGSC; http:// www.fgsc.net/) [56]. Double deletion strains (such as $\Delta N c a p 3 m \Delta N c a p 3 b)$ were generated by performing sexual crosses following previously described protocols [57]. The genotypes of single and double deletion strains were confirmed by PCR as described in Wu et al. [58] using the primers listed in Additional file 4: Table S1. The QM6a strain of T. reesei was kindly donated by Dr. Monika Schmoll (Department of Health and EnvironmentBioresources, Austrian Institute of Technology).

\section{Culture conditions and screens for $N$. crassa KO mutants}

Neurospora crassa stock cultures were maintained on minimal medium agar slants containing $1 \times$ Vogel's salt with $2 \%(\mathrm{w} / \mathrm{v})$ sucrose [59] at $25^{\circ} \mathrm{C}$. For enzyme production, 10-day-old conidia of $N$. crassa strains were suspended in sterile water and inoculated using a final concentration of $1 \times 10^{6}$ per mL into a 250 -mL Erlenmeyer flask containing $100 \mathrm{~mL}$ medium [60] [1× Vogel's salt, 2\% w/v crystalline cellulose (Avicel PH-101; SigmaAldrich, St. Louis, MO, USA), and $0.75 \% \mathrm{w} / \mathrm{v}$ yeast extract (Sigma-Aldrich)]. Flask batch culturing was performed at $25^{\circ} \mathrm{C}$ under constant light and with agitation $(200 \mathrm{rpm})$ for 7 days; extracellular protein titers were then measured based on the Bradford method. For secreted protein assays, two biological replicates were 


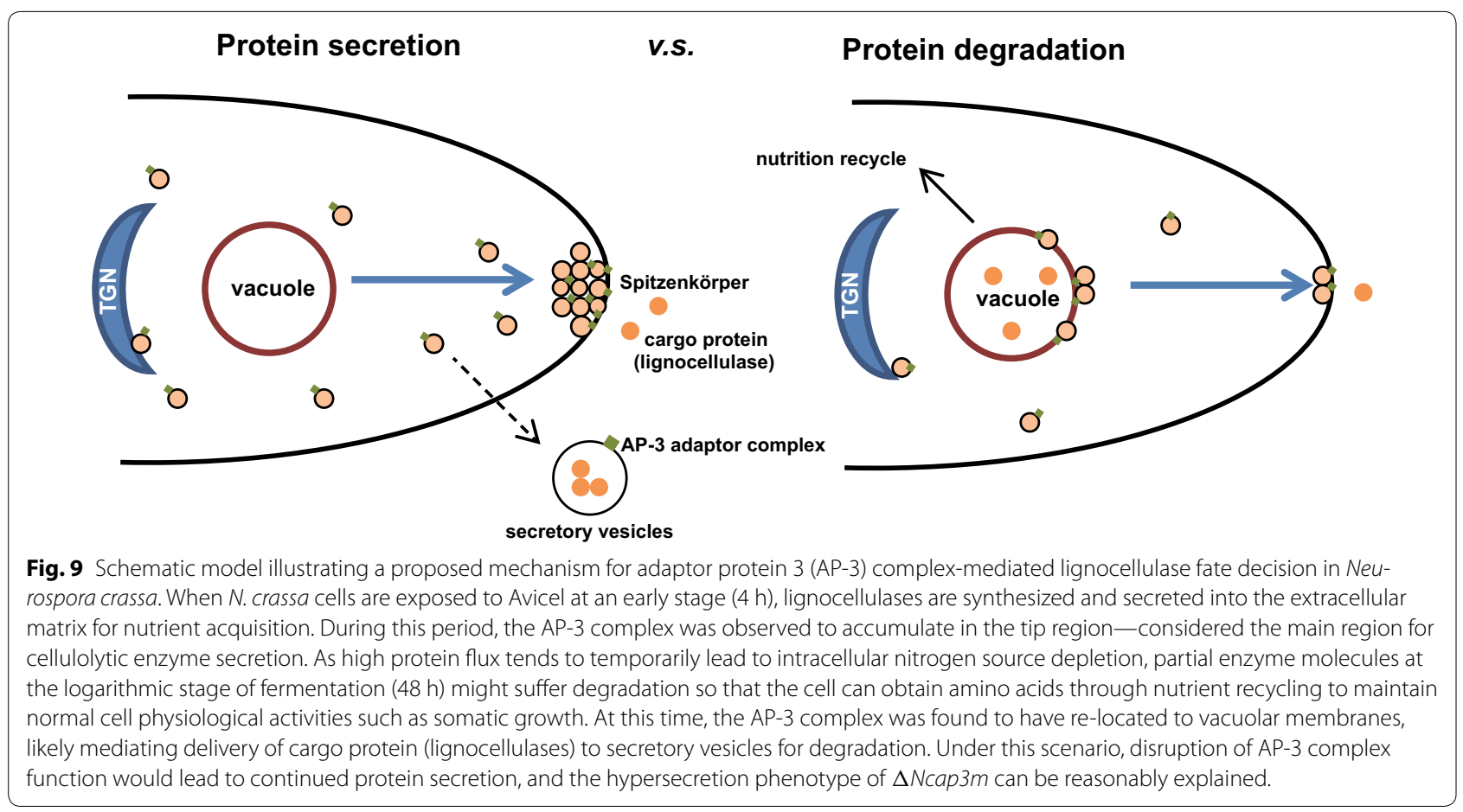

cultured per strain and three technical replicates were conducted per culture.

\section{Identification of orthologs and phylogenetic analysis}

The protein sequences of genes with potential malfunctions due to various mutations of $T$. reesei RUTC30 were extracted from the latest genome data maintained by the DOE Joint Genome Institute (http://genome.jgi.doe.gov/ TrireRUTC30_1/TrireRUTC30_1.home.html) using Perl scripts. Homologs of $N$. crassa proteins were identified using local BLASTp (version Blast+ 2.2.28) with an $E$ value $<10^{-5}$ applied as a cutoff. Phylogenetic analysis of Ncap3 $m$ was carried out in MEGA6 using the maximum likelihood method with 1,000 bootstrap replicates. Database homology searching was performed with the local BLAST program as described above.

\section{Complementation of $\Delta \mathbf{N c a p} 3 \mathrm{~m}$ in $\mathbf{N}$. crassa}

Complementary plasmids containing the Ncap3m coding region under the control of native or strong promoters were constructed separately. For the former case, the 1,566-bp full-length ORF of Ncap3m without a stop codon and with a 1,000-bp upstream putative native promoter region was PCR-amplified from WT N. crassa genomic DNA using the primers Pnative-F and ap3m$\mathrm{R}$ (Additional file 4: Table S1). After digestion with NotI and PacI, the fragment was inserted into plasmid pMF272 [61] and the resulting plasmid was designated as Pn-ap3m. For the latter case, the 1,566-bp ORF region of
Ncap3m was PCR amplified from WT N. crassa genomic DNA using the primer set ap3m-F/R (Additional file 4: Table S1), digested with $X b a \mathrm{I}$ and PacI, and then cloned into the downstream $c c g-1$ promoter within plasmid pMF272. The resulting plasmid was designated as Pcap $3 m$. Complementary plasmids were transformed into the host strain with a double deletion ( $\Delta$ Ncap3m:his3-) according to the method described in Margolin et al. (http://www.fgsc.net/fgn44/margol.html). Transformants were screened by selection for histidine prototrophy and green fluorescent protein fluorescence of conidia.

\section{T. reesei ap $3 m$ gene re-annotation and cloning}

For a heterologous functional complementation analysis, we conducted an inter-complementation assay by introducing the orthologs of NCU03998 in T. reesei into the ap $3 m$ mutant. The current ORF of tre53811 (the predicted ortholog of NCU03998) contained 3,658 bp with three introns and encoded a polypeptide of 1,007 amino acids containing three domains: a clathrin adaptor complex small chain, an AP-3 complex medium Mu3, and a malonyl CoA-acyl carrier protein transacylase (Additional file 1: Figure S1). We cloned the homologous gene cDNA of ap3m in T. reesei QM6a (in-house annotation, modified from jgi_Trire2_53811), consisting of 1,611 bp encoding a polypeptide of 536 amino acids containing the first two domains (amplified with primers Trap3m-F and Trap3m-R) (Additional file 4: Table S1, Additional file 2: Figure S2, Additional file 5: Figure S3). This gene under 
the control of the $c c g 1$ promoter was named Pc-Trap $3 m$ and then transformed into the host strain as described above to yield the selected transformant Pc-Trap $3 m$.

\section{RNA extraction}

Ten-day-old conidia of $N$. crassa strains were collected and inoculated into $1 \times$ Vogel's salt solution with $2 \%$ sucrose and grown for $16 \mathrm{~h}$. Mycelia were collected, washed several times with $1 \times$ Vogel's salt solution, transferred into medium with $2 \%$ Avicel or xylan as the carbon source, and grown to different time points $(4,48$, 96, or 168 h). Mycelia were harvested by vacuum filtration, frozen immediately in liquid nitrogen, and stored at $-80^{\circ} \mathrm{C}$ for RNA extraction. Total RNA from frozen samples was isolated using Trizol reagent (Invitrogen Life Technologies, Carlsbad, CA, USA) in accordance with the manufacturer's protocol. An additional clean-up was performed using an RNeasy mini kit (Qiagen, Hilden, Germany), according to the manufacturer's RNA Clear Up instructions. RNA integrity and concentration were checked on a Nanodrop instrument and by agarose gel electrophoresis.

\section{qPCR}

Quantitative real-time PCR was performed on a CFX96 real-time PCR detection system (Bio-Rad, Hercules, CA, USA) using reagents supplied with a Toyobo One-Step qPCR kit (Toyobo, Osaka, Japan). The 20- $\mu \mathrm{L}$ reaction mixtures, with three replicates, included 75-ng template RNA, $0.4 \mu \mathrm{M}$ primers, and 10- $\mu \mathrm{L}$ RNA-direct SYBR Green Real-Time PCR Master Mix. The relative transcript level of each gene was calculated by the $2^{-\Delta \Delta C_{t}}$ method, with its expression in the WT strain used as the control and the expression of the actin gene (NCU04173) used as an internal standard for all experiments. Specific primers for qPCR are listed in Additional file 4: Table S1.

\section{Protein and enzyme activity measurements}

Total extracellular protein content in supernatants was measured using a Bio-Rad DC protein assay kit (BioRad) based on absorbance at $595 \mathrm{~nm}$, with bovine serum albumin used as the standard. FPA was measured by the 3,5-dinitrosalicylic acid method [62]. Exoglucanase activity was measured at $50^{\circ} \mathrm{C}$ using $1.0 \mathrm{mg} / \mathrm{mL} p$-nitrophenyl$\beta$-D-cellobioside (Sigma-Aldrich) in $50 \mathrm{mM}$ citrate buffer ( $\mathrm{pH} 4.8)$ as the substrate. The reaction mixture containing $250 \mu \mathrm{L}$ of properly diluted enzyme and $250 \mu \mathrm{L}$ of $1.0 \mathrm{mg} / \mathrm{mL}$ substrate in $50 \mathrm{mM}$ citrate buffer $(\mathrm{pH} 4.8)$ was incubated for $10 \mathrm{~min}$ at $50^{\circ} \mathrm{C}$, and the reaction was terminated by the addition of $500 \mu \mathrm{L}$ of $1 \mathrm{M} \mathrm{Na}_{2} \mathrm{CO}_{3}$. The release of $p$-nitrophenol ( $p \mathrm{NP})$ was monitored at an absorbance at $420 \mathrm{~nm}$. The control was the inactivated enzyme, which was boiled at $100^{\circ} \mathrm{C}$ for $10 \mathrm{~min}$. $p \mathrm{NP}$ was used to generate a standard curve. In exoglucanase activity analyses, one unit of enzymatic activity was defined as the amount of $p \mathrm{NP}$ released from the substrate per minute using $1 \mathrm{~mL}$ enzyme under the standard assay conditions. Endoglucanase activity in culture supernatants was determined using an azo-cm-cellulose assay kit (Megazyme, Wicklow, Ireland) as described by the manufacturer. Endo-1,4- $\beta$-xylanase activities were assayed using an azo-xylan kit (Megazyme) according to the manufacturer's instructions.

\section{Fluorescence microscopy and image processing}

To localize NcAP3m-EGFP fusion protein using microscopy, the complemented $\Delta N c a p 3 m:: N c a p 3 m-E G F P$ strain was inoculated into liquid minimal medium and grown for $16 \mathrm{~h}$. The hyphae were harvested, washed with Vogel's salt solution, and transferred into inducing medium containing $0.5 \%(\mathrm{w} / \mathrm{v})$ Avicel for another 4 or $48 \mathrm{~h}$. EGFP fluorescence observations were performed on an Olympus BX51 fluorescence microscopy system. To co-localize the Spitzenkörper, $\Delta$ Ncap3m::Ncap3mEGFP cells were also stained with red-fluorescent FM464 dye (Invitrogen) at a final concentration of $10 \mu \mathrm{M}$ in Hanks' balanced salt solution buffer following the manufacturer's instructions.

\section{Statistical analyses}

Unless otherwise noted, all experiments were performed in triplicate and statistical tests for significance were determined via one-way analysis of variance using $R$ (version 3.1.1).

\section{Additional files}

Additional file 1: Figure S1. Protein sequence of tre53811 from Trichoderma reesei QM6a.

Additional file 2: Figure S2. Diagram of tre2_53811 protein from Trichoderma reesei QM6a.

Additional file 3: Figure S4. Phylogenetic tree of alkaline phosphatase proteins.

Additional file 4: Table S1. Primers used in this study.

Additional file 5: Figure S3. Protein sequence of the in-house-annotated AP-3 $\mu$ subunit from Trichoderma reesei QM6a.

\section{Abbreviations \\ ALP: alkaline phosphatase; AP-3: adaptor protein complex 3; bp: base pair; CCR: carbon catabolite repression; EGFP: enhanced green fluorescent protein; FM4-64: N-(3-triethylammoniumpropyl)-4-(6-(4(diethylamino) phenyl) hex- atrienyl) pyridinium dibromide; FPA: filter paper activity; KO: knockout; ORF: open reading frame; $p N P$ : $p$-nitrophenol; qPCR: quantitative real-time PCR; RESS: repression under secretion stress; WT: wild type.}

Authors' contributions

$X P, F F, L L, S Z$, and $C T$ designed the study and $X P, F F, L L, W S$, and $Y C$ performed the research. $X P, F F$, and $L L$ analyzed the data and $X P, F F, S Z$, and $C T$ wrote the paper. All authors read and approved the final manuscript. 


\section{Author details}

${ }^{1}$ College of Plant Sciences, Jilin University, Changchun 130062, China. ${ }^{2}$ Key Laboratory of Systems Microbial Biotechnology, Tianjin Institute of Industrial Biotechnology, Chinese Academy of Sciences, Tianjin 300308, China.

\section{Acknowledgements}

This work was supported by a Grant from the 973 Program of China (2011CB707403 and 2011CBA00803) and the 863 Project (2012AA022203D and SS2014AA021300). The authors wish to thank Zhiyong Sun, Guoli Ma, and Huiyan Li for help with the experiments and for critical reading of the manuscript.

\section{Compliance with ethical guidelines}

\section{Competing interests}

The authors declare that they have no competing interests.

Received: 19 June 2015 Accepted: 30 July 2015

Published online: 20 August 2015

\section{References}

1. Somerville C, Bauer S, Brininstool G, Facette M, Hamann T, Milne J et al (2004) Toward a systems approach to understanding plant-cell walls. Science 306(5705):2206-2211

2. Glass NL, Schmoll M, Cate JHD, Coradetti S (2013) Plant cell wall deconstruction by ascomycete fungi. Annu Rev Microbiol 67:477-498

3. Himmel ME, Ding SY, Johnson DK, Adney WS, Nimlos MR, Brady JW et al (2007) Biomass recalcitrance: engineering plants and enzymes for biofuels production. Science 315(5813):804-807

4. Cheng Y, Song X, Qin Y, Qu Y (2009) Genome shuffling improves production of cellulase by Penicillium decumbens JU-A10. J Appl Microbiol 107(6):1837-1846

5. Glenn M, Ghosh A, Ghosh BK (1985) Subcellular fractionation of a hypercellulolytic mutant, Trichoderma reesei Rut-C30-localization of endoglucanase in microsomal fraction. Appl Environ Microbiol 50(5):1137-1143

6. Yan SM, Wu G (2013) Secretory pathway of cellulase: a mini-review. Biotechnol Biofuels 6:177

7. Mcgavin M, Lam J, Forsberg CW (1990) Regulation and distribution of Fibrobacter succinogenes subsp. succinogenes $\mathbf{5} 85$ endoglucanases. App Environ Microbiol 56(5):1235-1244

8. Conesa A, Punt PJ, van Luijk N, van den Hondel CAMJJ (2001) The secretion pathway in filamentous fungi: a biotechnological view. Fungal Genet Biol 33(3):155-171

9. Zhang FX, Paterson AJ, Huang P, Wang K, Kudlow JE (2007) Metabolic control of proteasome function. Physiology 22(6):373-379

10. Li SC, Kane PM (2009) The yeast lysosome-like vacuole: endpoint and crossroads. BBA Mol Cell Res 1793(4):650-663

11. Peterson R, Nevalainen H (2012) Trichoderma reesei RUT-C30_thirty years of strain improvement. Microbiology 158(Pt 1):58-68

12. Bisaria VS, Ghose TK (1981) Biodegradation of cellulosic materials-substrates, microorganisms, enzymes and products. Enzyme Microb Technol 3(2):90-104

13. Montenecourt BS, Eveleigh DE (1977) Semiquantitative plate assay for determination of cellulase production by Trichoderma viride. Appl Environ Microbiol 33(1):178-183

14. Montenecourt BS, Eveleigh DE (1977) Preparation of mutants of Trichoderma reesei with enhanced cellulase production. Appl Environ Microbiol 34(6):777-782

15. Cherry JR, Fidantsef AL (2003) Directed evolution of industrial enzymes: an update. Curr Opin Biotech 14(4):438-443

16. Ilmén M, Thrane C, Penttilä M (1996) The glucose repressor gene cre 1 of Trichoderma: isolation and expression of a full-length and a truncated mutant form. Mol Gen Genet 251(4):451-461

17. Geysens S, Pakula T, Uusitalo J, Dewerte I, Penttila M, Contreras R (2005) Cloning and characterization of the glucosidase II alpha subunit gene of Trichoderma reesei: a frameshift mutation results in the aberrant glycosylation profile of the hypercellulolytic strain Rut-C30. Appl Environ Microbiol 71(6):2910-2924
18. Vitikainen M, Arvas M, Pakula T, Oja M, Penttila M, Saloheimo M (2010) Array comparative genomic hybridization analysis of Trichoderma reesei strains with enhanced cellulase production properties. BMC Genom 11:441

19. Le Crom S, Schackwitz W, Pennacchio L, Magnuson JK, Culley DE, Collett JR et al (2009) Tracking the roots of cellulase hyperproduction by the fungus Trichoderma reesei using massively parallel DNA sequencing. Proc Natl Acad Sci USA 106(38):16151-16156

20. Poggi-Parodi D, Bidard F, Pirayre A, Portnoy T, Blugeon C, Seiboth B et al (2014) Kinetic transcriptome analysis reveals an essentially intact induction system in a cellulase hyper-producer Trichoderma reesei strain. Biotechnol Biofuels 7:173

21. Znameroski EA, Glass NL (2013) Using a model filamentous fungus to unravel mechanisms of lignocellulose deconstruction. Biotechnol Biofuels 6(1):6

22. Sun J, Glass NL (2011) Identification of the CRE-1 Cellulolytic Regulon in Neurospora crassa. PLoS One 6(9):e25654. doi:10.1371/journal.pone.0025654

23. Tian C, Beeson WT, lavarone AT, Sun J, Marletta MA, Cate JH et al (2009) Systems analysis of plant cell wall degradation by the model filamentous fungus Neurospora crassa. Proc Natl Acad Sci USA 106(52):22157-22162

24. Phillips CM, lavarone AT, Marletta MA (2011) Quantitative proteomic approach for cellulose degradation by Neurospora crassa. J Proteome Res 10(9):4177-4185

25. Feraru E, PaciorekT, Feraru MI, Zwiewka M, De Groodt R, De Rycke R et al (2010) The AP-3 $\beta$ adaptin mediates the biogenesis and function of lytic vacuoles in Arabidopsis. Plant Cell 22(8):2812-2824

26. Robinson MS, Bonifacino JS (2001) Adaptor-related proteins. Curr opin Cell Biol 13:10

27. Sun JP, Tian CG, Diamond S, Glass NL (2012) Deciphering transcriptional regulatory mechanisms associated with hemicellulose degradation in Neurospora crassa. Eukaryot Cell 11(4):482-493

28. Freitag M, Hickey PC, Raju NB, Selker EU, Read ND (2004) GFP as a tool to analyze the organization, dynamics and function of nuclei and microtubules in Neurospora crassa. Fungal Genet Biol 41(10):897-910

29. Harris SD (2009) The Spitzenkorper: a signalling hub for the control of fungal development? Mol Microbiol 73(5):733-736

30. Cowles CR, Odorizzi G, Payne GS, Emr SD (1997) The AP-3 adaptor complex is essential for cargo-selective transport to the yeast vacuole. Cell 91(1):109-118

31. Zwiewka M, Feraru E, Moller B, Hwang I, Feraru MI, Kleine-Vehn J et al (2011) The AP-3 adaptor complex is required for vacuolar function in Arabidopsis. Cell Res 21(12):1711-1722

32. Fan FY, Ma GL, Li JG, Liu Q, Benz JP, Tian CG et al (2015) Genome-wide analysis of the endoplasmic reticulum stress response during lignocellulase production in Neurospora crassa. Biotechnol Biofuels 8:66

33. Gasser B, Saloheimo M, Rinas U, Dragosits M, Rodriguez-Carmona E, Baumann Ket al (2008) Protein folding and conformational stress in microbial cells producing recombinant proteins: a host comparative overview. Microb Cell Fact 7:11

34. Martinez IM, Chrispeels MJ (2003) Genomic analysis of the unfolded protein response in Arabidopsis shows its connection to important cellular processes. Plant Cell 15(2):561-576

35. Pakula TM, Laxell M, Huuskonen A, Uusitalo J, Saloheimo M, Penttila M (2003) The effects of drugs inhibiting protein secretion in the filamentous fungus Trichoderma reesei-evidence for down-regulation of genes that encode secreted proteins in the stressed cells. J Biol Chem 278(45):45011-45020

36. Fagundes MR, Lima JF, Savoldi M, Malavazi I, Larson RE, Goldman MH et al (2004) The Aspergillus nidulans npkA gene encodes a Cdc2-related kinase that genetically interacts with the UvsBATR kinase. Genetics 167(4):1629-1641

37. Pebernard S, Perry JJP, Tainer JA, Boddy MN (2008) Nse1 RING-like domain supports functions of the Smc5-Smc6 holocomplex in genome stability. Mol Biol Cell 19(10):4099-4109

38. Pakula TM, Salonen K, Uusitalo J, Penttila M (2005) The effect of specific growth rate on protein synthesis and secretion in the filamentous fungus Trichoderma reesei. Microbiology 151:135-143

39. Soulard A, Lechler T, Spiridonov V, Shevchenko A, Shevchenko A, Li R et al (2002) Saccharomyces cerevisiae Bzz1p is implicated with type I myosins in actin patch polarization and is able to recruit actin-polymerizing machinery in vitro. Mol Cell Biol 22(22):7889-7906 
40. Wendland J, Walther A (2006) Tip growth and endocytosis in fungi. Plant Endocytosis 1:8

41. Boehm M, Bonifacino JS (2002) Genetic analyses of adaptin function from yeast to mammals. Gene 286(2):175-186

42. Dell'Angelica EC (2009) AP-3-dependent trafficking and disease: the first decade. Curr Opin Cell Biol 21(4):552-559

43. Hirst J, Barlow LD, Francisco GC, Sahlender DA, Seaman MNJ, Dacks JB et al (2011) The fifth adaptor protein complex. PLoS Biol 9(10):e1001170

44. Mullins C, Hartnell LM, Bonifacino JS (2000) Distinct requirements for the AP-3 adaptor complex in pigment granule and synaptic vesicle biogenesis in Drosophila melanogaster. Mol Gen Genet 263(6):1003-1014

45. DellAngelica EC, Ohno H, Ooi CE, Rabinovich E, Roche KW, Bonifacino JS (1997) AP-3: an adaptor-like protein complex with ubiquitous expression. EMBO J 16(5):917-928

46. Simpson F, Peden AA, Christopoulou L, Robinson MS (1997) Characterization of the adaptor-related protein complex, AP-3. J Cell Biol 137(4):835-845

47. Starr TL, Pagant S, Wang CW, Schekman R (2012) Sorting signals that mediate traffic of chitin synthase III between the TGN/endosomes and to the plasma membrane in yeast. PLoS One 7(10):e46386

48. Klionsky DJ, Emr SD (1989) Membrane-protein sorting-biosynthesis, transport and processing of yeast vacuolar alkaline-phosphatase. EMBO J 8(8):2241-2250

49. Noda T, Ohsumi Y (1998) Tor, a phosphatidylinositol kinase homologue, controls autophagy in yeast. J Biol Chem 273(7):3963-3966

50. Klionsky DJ (2007) Monitoring autophagy in yeast: the Pho8 $\Delta 60$ assay. Methods Mol Biol 390:363-371

51. Donella-Deana A, Ostojic S, Pinna LA, Barbaric S (1993) Specific dephosphorylation of phosphopeptides by the yeast alkaline-phosphatase encoded by Pho8 gene. Biochim Biophys Acta 1177(2):221-228
52. Dai XY, Zhao MM, Cai Y, Guan QC, Zhao Y, Guan YF et al (2013) Phosphateinduced autophagy counteracts vascular calcification by reducing matrix vesicle release. Kidney Int 83(6):1042-1051

53. Cate AR, Syrbu S (1974) A relationship between alkaline phosphatase activity and the phagocytosis and degradation of collagen by the fibroblast. J Anat 117(Pt 2):351-359

54. Miyabe S, Izawa S, Inoue Y (2001) The Zrc1 is involved in zinc transport system between vacuole and cytosol in Saccharomyces cerevisiae. Biochem Bioph Res Commun 282(1):79-83

55. Qiao W, Ellis C, Steffen J, Wu CY, Eide DJ (2009) Zinc status and vacuolar zinc transporters control alkaline phosphatase accumulation and activity in Saccharomyces cerevisiae. Mol Microbiol 72(2):320-334

56. McCluskey K (2003) The fungal genetics stock center: from molds to molecules. Adv Appl Microbiol 52:245-262

57. Cai PL, Wang B, Ji JX, Jiang YS, Wan L, Tian CG et al (2015) The putative cellodextrin transporter-like protein CLP1 Is involved in cellulase induction in Neurospora crassa. J Biol Chem 290(2):788-796

58. Wu W, Hildebrand A, Kasuga T, Xiong X, Fan Z (2013) Direct cellobiose production from cellulose using sextuple $\beta$-glucosidase gene deletion Neurospora crassa mutants. Enzyme Microb Technol 52(3):184-189

59. Vogel HJ (1956) A convenient growth medium for Neurospora (Medium N). Microbiol Genet Bull 13:5

60. Yazdi MT, Radford A, Keen JN, Woodward JR (1990) Cellulase production by Neurospora Crassa - purification and characterization of cellulolytic enzymes. Enzyme Microb Technol 12(2):120-123

61. Folco HD, Freitag M, Ramon A, Temporini ED, Alvarez ME, Garcia I et al (2003) Histone $\mathrm{H} 1$ is required for proper regulation of pyruvate decarboxylase gene expression in Neurospora crassa. Eukaryot Cell 2(2):341-350

62. Ghose TK (1987) Measurement of cellulase activities. Pure Appl Chem 59(2):257-268

\section{Submit your next manuscript to BioMed Central and take full advantage of:}

- Convenient online submission

- Thorough peer review

- No space constraints or color figure charges

- Immediate publication on acceptance

- Inclusion in PubMed, CAS, Scopus and Google Scholar

- Research which is freely available for redistribution

Submit your manuscript at

www.biomedcentral.com/submit

C Biomed Central 